\title{
Wideband spectrum sensing based on serial multi-coset sampling for cognitive radio
}

\author{
Changjian Liu, Wuhuang Huang ${ }^{\mathrm{a}}$, and Houjun Wang \\ School of Automation Engineering, \\ University of Electronic Science and Technology of China, \\ No. 2006 Xiyuan Ave, West Hi-Tech Zone, Chengdu, 611731, China
}

a)huangwuhuang@163.com

\begin{abstract}
In this letter, a wideband spectrum sensing method is proposed for cognitive radio. Firstly, a serial Multi-Coset Sampling (MCS) structure is designed to avoid the mismatch among sub-Analog-to-Digital Converters (ADC) in traditional parallel MCS. Moreover, a five-step spectrum sensing method is presented based on finite resolution power spectral density estimation and energy detection. Finally, sampling pattern design is proved to be a minimal circular sparse ruler problem with an additional constraint. Simulations show that the serial MCS exhibits outstanding detection performance for signals with SNR higher than $-3 \mathrm{~dB}$ when the number of samples per coset is greater than 1400 .
\end{abstract}

Keywords: multi-coset sampling, spectrum sensing, sparse ruler Classification: Devices, circuits and systems for IoT and biomedical applications

\section{References}

[1] T. Yucek, et al.: "A survey of spectrum sensing algorithms for cognitive radio applications,” IEEE Commun. Surv. Tutorials 11 (2009) 116 (DOI: 10.1109/ SURV.2009.090109).

[2] M. Mishali, et al.: "From theory to practice: Sub-Nyquist sampling of sparse wideband analog signals," IEEE J. Sel. Topics Signal Process. 4 (2010) 375 (DOI: 10.1109/JSTSP.2010.2042414).

[3] M. Mishali, et al.: "Blind multiband signal reconstruction: compressed sensing for analog signals,” IEEE Trans. Signal Process. 57 (2009) 993 (DOI: 10.1109/ TSP.2009.2012791).

[4] E. P. Yang, et al.: "Modulated wideband converter with run length limited sequences," IEICE Electron. Express 13 (2016) 20160670 (DOI: 10.1587/elex. 13.20160670).

[5] K. Cao, et al:: "A novel sub-Nyquist multiband signal detection algorithm for cognitive radio," submitted to IEEE Trans. Signal Process.

[6] H. H. Chen, et al.: "Digital background calibration for timing mismatch in time-interleaved ADCs," Electron. Lett. 42 (2006) 74 (DOI: 10.1049/ el:20063387).

[7] K. J. Yang, et al.: "Timing skew calibration method for TIADC-based 20 GSPS digital storage oscilloscope,” J. Circuits Syst. Comput. 25 (2016) 1650007 (DOI: 10.1142/S0218126616500079). 
[8] M. Wakin, et al.: "A nonuniform sampler for wideband spectrally-sparse environments," IEEE J. Emerg. Sel. Topics Circuits Syst. 2 (2012) 516 (DOI: 10.1109/JETCAS.2012.2214635).

[9] R. Grigoryan, et al:: "Computational complexity reduction in nonuniform compressed sensing by multi-coset emulation," Signal Process. 131 (2017) 492 (DOI: 10.1016/j.sigpro.2016.04.004).

[10] C. P. Yen, et al.: "Wideband spectrum sensing based on sub-Nyquist sampling,” IEEE Trans. Signal Process. 61 (2013) 3028 (DOI: 10.1109/TSP. 2013.2251342).

[11] W. Sun, et al.: "Wideband power spectrum sensing and reconstruction based on single channel sub-Nyquist sampling," IEICE Trans. Fundamentals 99.A (2016) 167 (DOI: 10.1587/transfun.E99.A.167).

[12] M. A. Lexa, et al.: "Compressive power spectral density estimation," IEEE ICASSP (2011) 3884 (DOI: 10.1109/ICASSP.2011.5947200).

[13] M. Shaghaghi, et al:: "Finite-length and asymptotic analysis of averaged correlogram for undersampled data," Appl. Comput. Harmon. Anal. 43 (2017) 404 (DOI: 10.1016/j.acha.2016.02.001).

[14] T. O. Dickson, et al:: "An 80-Gb/s 2/sup 31/-1 pseudorandom binary sequence generator in SiGe BiCMOS technology," IEEE J. Solid-State Circuits 40 (2005) 2735 (DOI: 10.1109/JSSC.2005.856578).

[15] Z. J. Qin, et al.: "Wideband spectrum sensing on real-time signals at subNyquist sampling rates in single and cooperative multiple nodes," IEEE Trans. Signal Process. 64 (2016) 3106 (DOI: 10.1109/TSP.2015.2512562).

\section{Introduction}

Spectrum sensing is an important task in cognitive radio, and its purpose is to detect whether primary users occupy the spectrum resources allocated to them. Spectrum bands that are not occupied by primary users are called spectrum holes. Secondary users can use these spectrum holes to transmit information in order to improve spectrum utilization efficiency. The well-known Nyquist sampling theorem states that alias-free sampling requires the sampling rate to be at least twice the maximum frequency of the signal. In practical wideband spectrum sensing, the available Analog-to-Digital Converters (ADC) often cannot satisfy the high sampling rate requirement for wideband signals. Even with such a high sampling rate ADC, the cost is usually quite high in terms of price and power consumption [1]. Therefore, it is of significant importance to study spectrum sensing based on sub-Nyquist techniques.

Several parallel sub-Nyquist sampling methods have emerged in recent years, such as Modulated Wideband Converter (MWC) [2] and parallel Multi-Coset Sampling (MCS) [3]. The input bandwidth of MWC is improved by implementing Run Length Limited (RLL) sequence under a certain chip rate [4]. However, it is difficult to suppress the unwanted harmonics due to the usage of Pseudo-Random Binary Sequence (PRBS) instead of a single-tone as the local oscillator signal. Another type of parallel sampling method is the parallel MCS in which several sub-ADCs work parallelly. The sampling clocks of these sub-ADCs have the same frequency but different phases. Parallel MCS can be implemented on a TimeInterleaved ADC (TIADC) platform to further improve its time resolution. Re- 
cently, a sparse multiband signal detector is proposed based on parallel MCS structure by utilizing the linear relation between the locations of nonzero frequencies and the Fourier Transform of the undersampled signals [5]. As mismatch among sub-ADCs, mainly offset mismatch, gain mismatch, and sampling clock skew, degrades the Spurious Free Dynamic Range (SFDR) of a TIADC system $[6,7]$, the mismatch can also severely impact the spectrum sensing performance of sampling structure based on parallel MCS.

The mismatch problem in parallel sampling structure can be avoided in singlechannel sampling structure. A single-channel non-uniform sampling (NUS) structure is proposed, and it mainly consists of a custom-designed front-end sampling chip and a commercial ADC [8]. A nonlinear compressive sensing algorithm is used to reconstruct the input signal. The reconstruction complexity is reduced by emulating MCS in NUS [9]. The custom-designed front-end sampling chip in NUS consists of two Sample/Holds $(\mathrm{S} / \mathrm{H})$, one is the master $\mathrm{S} / \mathrm{H}$ and the other is the slave $\mathrm{S} / \mathrm{H}$.

The above mentioned sub-Nyquist sampling methods $[2,3,4,8,9]$ focus on signal reconstruction, while one may only be interested in the power spectrum of signal in some practical applications, such as cognitive radio and spectrum monitoring. The Power Spectral Density (PSD) of signal is directly estimated using sub-Nyquist samples by exploiting the statistical property of signal [10]. A single channel sub-Nyquist sampling system is also designed for PSD estimation [11]. In [12], the statistical property of signal is also used to estimate the finite resolution PSD without requiring the signal to be sparse. The mean and variance of finite resolution PSD estimator are investigated when the number of sub-Nyquist samples is limited [13]. However, the finite resolution PSD estimator proposed in [12] is based on parallel MCS, and the mismatch among sub-ADCs will have great adverse impact on the spectrum sensing performance.

In this letter, a novel wideband spectrum sensing method is proposed for cognitive radio. This method is based on single-channel sub-Nyquist sampling structure but without sparsity requirement. Firstly, a single-channel sub-Nyquist sampling structure is designed and we name it serial MCS. This structure shifts the high sampling rate burden for wideband signals from $\mathrm{ADC}$ to $\mathrm{S} / \mathrm{H}$. Compared with the NUS $[8,9]$, the proposed serial MCS scheme uses only one $\mathrm{S} / \mathrm{H}$, thus the power consumption of the sampling circuit can be reduced. Moreover, a spectrum sensing method is presented. The statistical property of signal is exploited to estimate the finite resolution PSD. The estimated results are further used to conduct spectrum sensing using energy detection method. Finally, sampling pattern is designed for the proposed serial MCS. The design is proved to be a minimal circular sparse ruler problem with an additional constraint. Simulations show that, in the proposed serial MCS, the primary users and spectrum holes can be detected with high probability for signals with SNR higher than $-3 \mathrm{~dB}$ when the number of samples per coset is greater than 1400 . 


\section{Serial multi-coset sampling}

An overview of the proposed serial MCS is shown in Fig. 1. The input signal is first sampled by $\mathrm{S} / \mathrm{H}$ and then captured by its following ADC. Two different periodic non-uniform sampling clocks are generated by the PRBS generator, and they are provided to $\mathrm{S} / \mathrm{H}$ and $\mathrm{ADC}$. The first periodic non-uniform clock is determined by the MCS sampling pattern, while the second periodic non-uniform clock is dependent on not only the sampling pattern but also the ADC's sampling rate. To begin with, the periodic non-uniformly sampled data are classified into several uniform sampling sequences. These uniform sampling sequences are further processed to construct a set of linear equations of finite resolution PSD. Then Least Square (LS) algorithm is used to estimate the finite resolution PSD. Eventually, the estimated finite resolution PSD is compared with a threshold to determine whether the spectrum cell is occupied or not.

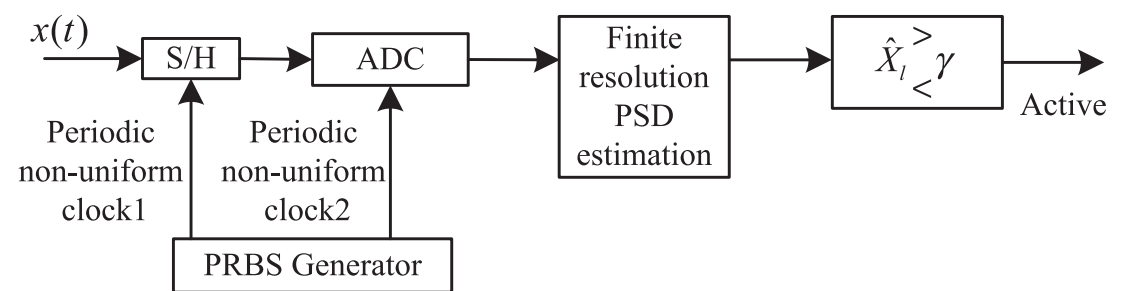

Fig. 1. The block diagram of serial MCS.

In detail, let $x(t)$ represent the input Wide-Sense Stationary (WSS) signal. $f_{N}$ and $T$ denote the signal's Nyquist sampling rate and interval, respectively. $x(t)$ is bandlimited to $\left[-f_{N} / 2, f_{N} / 2\right) . f_{A D C}$ and $T_{A D C}$ denote the signal's sampling rate and interval, respectively. Let $d$ represent the ratio between $f_{N}$ and $f_{A D C}, d=$ $f_{N} / f_{A D C}$. In MCS, $q$ samples are selected from $L$ consecutive Nyquist samples using the sampling pattern $C=\left\{c_{1}, c_{2}, \ldots, c_{q}\right\}$, where $1 \leq c_{1}<c_{2}<\cdots<c_{q} \leq L$ are the sample index. Let $N_{0}$ denote the number of samples per coset, and the total number of periodic non-uniformly sampled data is $N=q \times N_{0}$. We will illustrate in section 4 'Sampling pattern design' that the sampling pattern must have at least one pair of cosets with only $1 \times T$ time difference. The ADC, whose sampling interval $T_{\mathrm{ADC}}=d \times T$ is greater than $T$, cannot capture two cosets $c_{i}$ and $c_{i+1}$ with only $1 \times T$ time difference directly. The $\mathrm{S} / \mathrm{H}$ in Fig. 1 is necessary and its function is to temporarily store the second coset $c_{i+1}$ until the ADC has finished capturing the previous coset $c_{i}$.

Fig. 2 gives an example of the sampling process, where $q=6, L=24$, $f_{A D C}=f_{N} / 4$ and $C=\{1,2,9,12,14,18\}$. The first coset $C(1)$ can be sampled directly, while the second coset $C(2)$ has to be firstly sampled by the $\mathrm{S} / \mathrm{H}$ and then captured by its following $\mathrm{ADC}$ on the $5^{\text {th }}$ Nyquist time grid. So the third $\operatorname{coset} C(3)$ has to be greater than 5 , otherwise, it will lose the last coset $C(2)$. The cooperation between the first and the second periodic non-uniform clock ensures that two adjacent coset with only $1 \times T$ time difference can be captured by an ADC with sampling rate $f_{A D C}$ much lower than $f_{N}$, and this is the essence of the proposed serial MCS. 
Next, the periodic non-uniformly sampled data is classified into several uniform sampling sequences. Let $y[n], 1 \leq n \leq N$ be the periodic non-uniformly sampled data output from the ADC

$$
y[n]=\left[y_{c 1}[0], y_{c 2}[0], \ldots, y_{c q}[0], y_{c 1}[1], \ldots, y_{c q}\left[N_{0}-1\right]\right],
$$

where $y_{c i}[k], 1 \leq i \leq q, 0 \leq k \leq N_{0}-1$ is the sample obtained from the $k$-th $L$ length consecutive Nyquist sample block using sample index $C(i)$. Then periodic non-uniformly sampled data $y[n], 1 \leq n \leq N$ is classified into $q$ uniform sampling sequences

$$
y_{c i}[k]=y[k \times q+i], \quad 0 \leq k \leq N_{0}-1, \quad 1 \leq i \leq q .
$$

Let $\boldsymbol{y}_{c i}=\left[y_{c i}[0], y_{c i}[1], \ldots, y_{c i}\left[N_{0}-1\right]\right]^{T}$ represent the $i$-th uniform sampling sequence which corresponds to the $i$-th $\operatorname{coset} c_{i}$. The sampling rate and time offset of $\boldsymbol{y}_{c i}$ are $f_{N} / L$ and $c i \times T$, respectively. $q$ uniform sampling sequences can be written in matrix form concisely

$$
\boldsymbol{y}_{c}=\left[\boldsymbol{y}_{c 1}, \boldsymbol{y}_{c 2}, \ldots, \boldsymbol{y}_{c q}\right]^{\mathrm{T}} .
$$

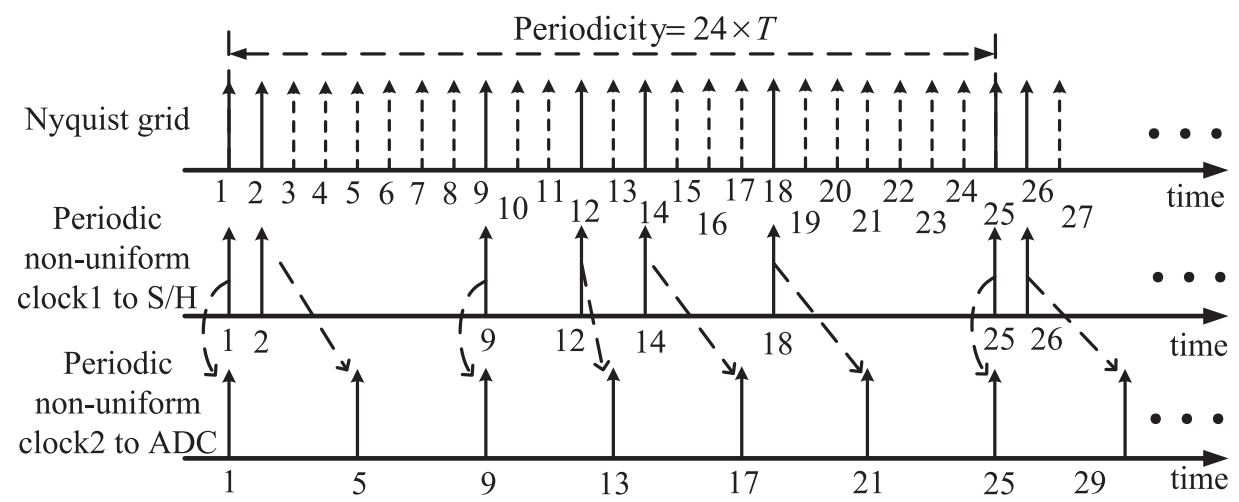

Fig. 2. The sampling process of serial MCS.

In practical implementation, the parameter of current available Integrated Circuits (IC) should be considered. The requirement for S/H's sampling rate is high, but the requirement for the following ADC's sampling rate is low. The proposed scheme shifts the high sampling rate burden from ADC to $\mathrm{S} / \mathrm{H}$. So the parameter of $\mathrm{S} / \mathrm{H}$ is very important to serial MCS. The sampling rate and input bandwidth of the current state-of-the-art S/H product are 4 Giga Samples Per Second (GSPS) and $18 \mathrm{GHz}$, respectively (HMC760LC4B, Analog Devices, Inc.). The jitter of the periodic non-uniform sampling clock is also an important parameter in serial MCS. The alternating rate and jitter of current state-of-the-art PRBS generator are $80 \mathrm{GHz}$ and $600 \mathrm{fs}$ (femtosecond), respectively [14]. Commercial parallel-to-serial converter can be used to generate the periodic non-uniform sampling clock, and the alternating rate and typical jitter of current state-of-art parallel-to-serial converter are $3.2 \mathrm{GHz}$ and $200 \mathrm{fs}$, respectively (MC100EP446, ON Semiconductor, Inc.). 


\section{Spectrum sensing}

After $q$ uniform sampling sequences have been obtained, a five-step spectrum sensing method is presented and summarized as follows:

1) Associate the aliasing spectrum with the original spectrum.

2) Calculate the covariance matrix.

3) Construct an overdetermined system of linear equations.

4) Estimate the finite resolution PSD.

5) Decision making.

1) Associate the aliasing spectrum with the original spectrum: Let $X(f)$ be the Fourier Transform of $x(t)$. The Discrete Time Fourier Transform (DTFT) of the $i$-th uniform sampling sequence $\boldsymbol{y}_{c i}$ can be written as

$$
\begin{aligned}
Y_{c i}\left(\mathrm{e}^{j 2 \pi f L T}\right) & =\sum_{n=-\infty}^{+\infty} y_{c i}[n] \mathrm{e}^{-j 2 \pi f n L T}, \quad f \in \mathcal{F}_{0} \\
& =\frac{1}{L T} \sum_{l=-\frac{L}{2}}^{\frac{L}{2}-1} X_{c i}\left(f-\frac{l}{L T}\right), \quad f \in \mathcal{F}_{0} \\
& =\frac{1}{L T} \sum_{l=-\frac{L}{2}}^{\frac{L}{2}-1} X\left(f-\frac{l}{L T}\right) \mathrm{e}^{j 2 \pi c_{i} T\left(f-\frac{l}{L T}\right)}, \quad f \in \mathcal{F}_{0} \\
& =\frac{1}{L T} \sum_{l=-\frac{L}{2}}^{\frac{L}{2}-1} X\left(f-\frac{l}{L T}\right) \mathrm{e}^{-j 2 \pi c_{i} \frac{l}{L}} \mathrm{e}^{j 2 \pi f c_{i} T}, \quad f \in \mathcal{F}_{0},
\end{aligned}
$$

where $\mathcal{F}_{0}=[0,1 /(L T))$ and $L$ is even. The bandlimited property $X(f)=0$ for $|f|>1 /(2 T)$ is used in the derivation from Eq. (4) to Eq. (5). The relationship between the delayed signal's spectrum and the original spectrum $X_{c i}(f)=$ $X(f) \mathrm{e}^{j 2 \pi f c_{i} T}$ is used in the derivation from Eq. (5) to Eq. (6). The term $\mathrm{e}^{j 2 \pi f c_{i} T}$ in Eq. (7) can be moved to the left of the equation, and define

$$
Y_{i}\left(\mathrm{e}^{j 2 \pi f L T}\right) \triangleq \mathrm{e}^{-j 2 \pi f c_{i} T} Y_{c i}\left(\mathrm{e}^{j 2 \pi f L T}\right) .
$$

Eq. (7) can be rewritten as

$$
Y_{i}\left(\mathrm{e}^{j 2 \pi f L T}\right)=\frac{1}{L T} \sum_{l=-\frac{L}{2}}^{\frac{L}{2}-1} X\left(f-\frac{l}{L T}\right) \mathrm{e}^{-j 2 \pi c_{i} \frac{l}{L}}, \quad f \in \mathcal{F}_{0} .
$$

The delay $c_{i} T$ in the $i$-th uniformly sampling sequence $\boldsymbol{y}_{c i}$ should be compensated before further processing. Fractional delay filters can be used to compensate the delay $c_{i} T$ which is smaller than $\boldsymbol{y}_{c i}$ 's sampling interval.

At this point, we have established the relationship between the aliasing spectrum and the original spectrum in Eq. (9) for single uniform sampling sequence $\boldsymbol{y}_{c i} . q$ uniform sampling sequences, or the entire MCS system, can be modeled in matrix form concisely

$$
\boldsymbol{Y}(f)=\boldsymbol{\Phi} \boldsymbol{x}(f), \quad f \in \mathcal{F}_{0}
$$

where $\boldsymbol{Y}_{i}(f)=Y_{i}\left(\mathrm{e}^{j 2 \pi f L T}\right), \boldsymbol{\Phi}_{i l}=\mathrm{e}^{-j 2 \pi c_{i} \frac{l}{L}}, \boldsymbol{x}_{l}(f)=X\left(f-\frac{l}{L T}\right), 1 \leq i \leq q$ and $-\frac{L}{2} \leq$ $l \leq \frac{L}{2}-1$.

2) Calculate the covariance matrix: In this part, the integral covariance matrix of $\boldsymbol{Y}(f)$ is explored and it can be written as

$$
\mathrm{R}_{\boldsymbol{Y}} \triangleq \int_{0}^{1 / L T} \mathrm{E} \boldsymbol{Y}(f) \boldsymbol{Y}(f)^{\mathrm{H}} d f,
$$




$$
\begin{aligned}
& =\boldsymbol{\Phi} \int_{0}^{1 / L T} \operatorname{E} \boldsymbol{x}(f) \boldsymbol{x}(f)^{\mathrm{H}} d f \boldsymbol{\Phi}^{\mathrm{H}}, \\
& =\boldsymbol{\Phi R}_{x} \boldsymbol{\Phi}^{\mathrm{H}},
\end{aligned}
$$

where

$$
\mathrm{R}_{x}=\int_{0}^{1 / L T} \operatorname{E} \boldsymbol{x}(f) \boldsymbol{x}(f)^{\mathrm{H}} d f .
$$

Since $x(t)$ is a WSS signal, the cross spectrum at two frequencies is $\mathrm{E} X\left(f_{1}\right) X\left(f_{2}\right)^{*}=\left|X\left(f_{1}\right)\right|^{2} \delta\left(f_{1}-f_{2}\right)$, where $\delta(\cdot)$ represents the kronecker delta function. So $\operatorname{E} \boldsymbol{x}(f) \boldsymbol{x}(f)^{\mathrm{H}}$ is a diagonal matrix for a specific frequency $f \in \mathcal{F}_{0}$, and its $l$-th diagonal element is $|X(f-l /(L T))|^{2}$. Similarly, $\mathrm{R}_{x}=$ $\int_{0}^{1 / L T} \operatorname{Ex}(f) \boldsymbol{x}(f)^{\mathrm{H}} d f$ is also a diagonal matrix and its $l$-th diagonal element is $X_{l}=\int_{0}^{1 / L T}|X(f-l /(L T))|^{2} d f$. In MCS, the original spectrum of $x(t)$, which is bandlimited to $\left[-f_{N} / 2, f_{N} / 2\right)$, is sliced into $L$ spectrum cells with width $f_{N} / L$. $X_{l}$ is the average power within the $l$-th spectrum cell, and it is called finite resolution PSD in [12]. Eq. (13) establishes the relationship between diagonal matrix $\mathrm{R}_{\boldsymbol{x}}$ and covariance $\mathrm{R}_{\boldsymbol{Y}}$, and it can be further written as

$$
\mathrm{R}_{\boldsymbol{Y}}(i, j)=\mathrm{E} \boldsymbol{Y}_{i}(f) \boldsymbol{Y}_{j}(f)^{\mathrm{H}}=\frac{1}{L T} \sum_{l=-\frac{L}{2}}^{\frac{L}{2}-1} X_{l} \mathrm{e}^{-j 2 \pi\left(c_{i}-c_{j}\right) \frac{l}{L}}, \quad 1 \leq i, j \leq q .
$$

Let $\overline{\boldsymbol{y}}_{c}$ be the time domain data after implementing fractional filters on $\boldsymbol{y}_{c}$. According to Parseval's theorem, $\mathrm{R}_{\boldsymbol{Y}}$ can be calculated as $\hat{\mathrm{R}}_{\boldsymbol{Y}}=\overline{\boldsymbol{y}}_{c} \overline{\boldsymbol{y}}_{c}{ }^{\mathrm{H}}$.

3) Construct an overdetermined system of linear equations: The $i$-th and $j$-th diagonal elements of sample covariance $\mathrm{R}_{Y}$, using Eq. (15), can be expressed as

$$
\begin{aligned}
\mathrm{R}_{\boldsymbol{Y}}(i, i) & =\frac{1}{L T} \sum_{l=-\frac{L}{2}}^{\frac{L}{2}-1} X_{l} \mathrm{e}^{j 0}, \\
\mathrm{R}_{\boldsymbol{Y}}(j, j) & =\frac{1}{L T} \sum_{l=-\frac{L}{2}}^{\frac{L}{2}-1} X_{l} \mathrm{e}^{j 0} .
\end{aligned}
$$

That is to say, all diagonal elements are equal and they can only construct a linear equation.

For non-diagonal elements, the elements in the upper and lower triangles are symmetrical and equal since the sample covariance $\mathrm{R}_{Y}$ is a real symmetric matrix. So the non-diagonal elements can only construct $\left(q^{2}-q\right) / 2$ equations. Let $i$ and $j$ represent the covariance matrix's row and column index, and define a new index $k \triangleq q(i-1)+j$. Let $u_{k}, 1 \leq k \leq\left(q^{2}-q\right) / 2$ represent the element $\mathrm{R}_{Y}(i, j)$ in the lower triangles of matrix $\mathrm{R}_{\boldsymbol{Y}}$ and it can be written as

$$
\boldsymbol{u}_{k} \triangleq \mathrm{R}_{\boldsymbol{Y}}(i, j)=\frac{1}{L T} \sum_{l=-\frac{L}{2}}^{\frac{L}{2}-1} X_{l} \mathrm{e}^{-j 2 \pi\left(c_{i}-c_{j}\right) \frac{l}{L}}, \quad 1 \leq j<i \leq q .
$$

The number of elements in the lower triangle of matrix $\mathrm{R}_{\boldsymbol{Y}}$ is $\left(q^{2}-q\right) / 2$ and each element can construct a linear equation. So $\left(q^{2}-q\right) / 2$ linear equations can be constructed, and they can be written in matrix form concisely

$$
\boldsymbol{u}=\Psi \boldsymbol{v},
$$

where $\boldsymbol{u}_{k}=\mathrm{R}_{\boldsymbol{Y}}(i, j), \quad 1 \leq k \leq\left(q^{2}-q\right) / 2, \quad \boldsymbol{v}_{l}=X_{l}, \quad-\frac{L}{2} \leq l \leq \frac{L}{2}-1$ and $\boldsymbol{\Psi}_{k l}=$ $\mathrm{e}^{-j 2 \pi\left(c_{i}-c_{j}\right) \frac{l}{L}}$. In addition, since the elements in matrix $\mathrm{R}_{Y}$ are real, the elements $\mathrm{e}^{-j 2 \pi\left(c_{i}-c_{j}\right) \frac{l}{L}}$ in Eq. (19) are complex, and the unknowns $X_{l}$ are also real, $\left(q^{2}-q\right) / 2$ 
complex equations can be viewed as $\left(q^{2}-q\right)$ real equations. Therefore, the total number of linear equations is $\left(q^{2}-q\right)+1$.

4) Estimate the finite resolution PSD: After building $q^{2}-q+1$ linear equations using the covariance matrix $R_{Y}$, we note that the number of unknowns in the system of linear equations is $L$. Here we assume $q^{2}-q+1 \geq L$ and the matrix $\boldsymbol{\Psi}$ has full column rank. For such an over-determined system, the LS algorithm can be used to estimate the finite resolution PSD. Note that PSD has non-negative property, so the Non-Negative LS (NNLS) algorithm can also be used to estimate the finite resolution PSD. NNLS imposes non-negative constraint on the over-determined system at the expense of additional computational cost. Higher estimation accuracy can be achieved using NNLS.

5) Decision making: After the finite resolution PSD is obtained, the estimated finite resolution PSD is compared with a predefined threshold to determine whether these spectrum cells are occupied or not. The predefined threshold $\gamma$ is determined by noise variance $\sigma^{2}$, false probability PF and the sample number $\mathbb{Z}$, and it can be written as

$$
\gamma=\sigma^{2}\left(1+\frac{Q^{-1}(\mathrm{PF})}{\sqrt{\mathbb{Z} / 2}}\right)
$$

where $Q^{-1}(\cdot)$ is the tail distribution function of the standard normal distribution [15]. In practical application, the noise variance $\sigma^{2}$ can be obtained from a certain spectrum band which is known for sure to be idle [15]. For example, the $21^{\text {st }}$ channel in TeleVision White Spaces (TVWS) should be idle in the UK at present [15]. If the finite resolution PSD is greater than the threshold $\gamma$, then it can be determined that the primary user is using the current spectrum cell. Otherwise, the spectrum cell is considered to be vacant, and the secondary user can use this spectrum cell to transmit its information.

\section{Sampling pattern design}

In the fourth step of the spectrum sensing method, we assumed that the matrix $\Psi$ has full column rank. In fact, whether it has full column rank depends on the design of the sampling pattern. Let us explore the structure of matrix $\Psi$. It can be written as the product of two matrices using Eq. (18) and Eq. (19),

$$
\Psi=\mathbf{E} \times \mathbf{F},
$$

where $\mathbf{F}$ is a $L \times L$ Discrete Fourier Transform (DFT) matrix and $\mathbf{E}$ is a $\left(q^{2}-q+1\right) \times L$ matrix. The row of matrix $\mathbf{E}$ contains only one non-zero element 1 and the other elements are zero, it acts like a selection vector which selects which row of $\mathbf{F}$ as the current row of $\boldsymbol{\Psi}$. The matrix $\mathbf{E}$ is also called selection matrix. Obviously, if the matrix $\mathbf{E}$ has full column rank, then matrix $\boldsymbol{\Psi}$ has full column rank since the DFT matrix $\mathbf{F}$ is a full rank square matrix.

A $L$-length circular sparse ruler can be viewed as a ruler with only $q$ marks which is less than $L$, but it is also able to measure all the length between 1 and $L$. Define $\Omega(C)=\left\{\left(c_{i}-c_{j}\right) \bmod L \mid \forall c_{i}, c_{j} \in C\right\}$, where $(\cdot \bmod \cdot)$ is the modulus operator and $C \subset\{1,2, \ldots, L\}$. A set $C$ is a circular sparse ruler if $\Omega(C)=$ $\{1,2, \ldots, L\}$. Fig. 3 gives an example of circular sparse ruler, where the measure- 
ment range $L=24$ and the marks are $C=\{1,2,9,12,14,18\}$. Although there is no mark 7 and 17 on the sparse ruler, length 7 and 17 can be measured using mark 2 and 9.

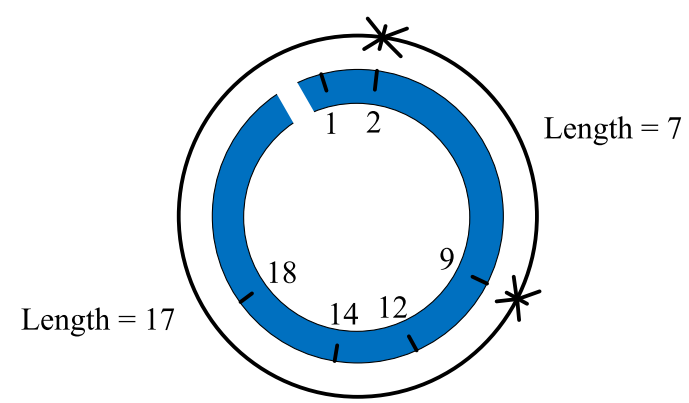

Fig. 3. A length-24 circular sparse ruler.

Note that the sample covariance matrix $\mathrm{R}_{Y}$ is a symmetric matrix, or $\mathrm{R}_{Y}(i, j)=\mathrm{R}_{Y}(j, i)$ for $1 \leq j<i \leq q$, so, using Eq. (18),

$$
\frac{1}{L T} \sum_{l=-\frac{L}{2}}^{\frac{L}{2}-1} X_{l} \mathrm{e}^{-j 2 \pi\left(c_{i}-c_{j}\right) \frac{l}{L}}=\frac{1}{L T} \sum_{l=-\frac{L}{2}}^{\frac{L}{2}-1} X_{l} \mathrm{e}^{-j 2 \pi\left(c_{j}-c_{i}\right) \frac{L}{L}},
$$

where $1 \leq j<i \leq q$. The term $\mathrm{e}^{-j 2 \pi\left(c_{j}-c_{i}\right) \frac{l}{L}}$ in Eq. (22) can be rewritten as $\mathrm{e}^{-j 2 \pi\left(c_{j}-c_{i}+L\right) \frac{l}{L}}$. That is to say, if the $\left(c_{i}-c_{j}\right)$-th row of DFT matrix $\mathbf{F}$ is selected, then $\left(c_{j}-c_{i}+L\right)$-th row of the DFT matrix $\mathbf{F}$ is also selected. The definition $\Omega(C)=\left\{\left(c_{i}-c_{j}\right) \bmod L \mid \forall c_{i}, c_{j} \in C\right\}$ can be rewritten as $\Omega(C)=\left\{\left(c_{i}-c_{j}\right)\right.$ or $\left.\left(c_{j}-c_{i}+L\right) \mid 1 \leq j<i \leq q\right\}$. If each element in $\{1,2, \ldots, L\}$ is included in $\Omega(C)$, or each column vector of matrix $\mathbf{E}$ contains at least one non-zero element 1 , combined with the fact that the row vector of $\mathbf{E}$ has only one non-zero element 1, the matrix $\mathbf{E}$ will have full column rank. So the sampling pattern design can be viewed as a circular sparse ruler problem. Circular sparse ruler uses the difference between two marks to measure any length between 1 and $L$. If we want to measure length 1 to length $L$, then at least one pair of marks, or cosets, should have length 1 difference, or $1 \times T$ time difference. Further, to obtain the strongest compression rate, we would like to minimize the cardinality of $C$, which can be viewed as a minimal circular sparse ruler problem. The last but not least, let $c_{i+2}$ and $c_{i}$ denote the $i+2$-th and $i$-th coset, respectively. $c_{i+2}$ should be greater than $c_{i}+d$ because the $\mathrm{S} / \mathrm{H}$ in Fig. 1 can only temporarily store one sample and the minimal sampling interval of ADC is $T_{A D C}=d \times T$. So the design of sampling pattern turns into the following optimization problem

$$
\min _{C}|C| \text { s.t. }\left\{\begin{array}{l}
\Omega(C)=\{1,2, \ldots, L\} \\
c_{i+2}>c_{i}+d, \quad 1 \leq i \leq q-2
\end{array},\right.
$$

where the sampling pattern $C \subset\{1,2, \ldots, L\}$.

\section{Simulation results}

The WSS signal which is used to test the proposed spectrum sensing method is generated by the following model: 


$$
x(t)=\sum_{i=1}^{M} s_{i}(t) \cos \left(2 \pi f_{i} t\right)+n(t),
$$

where $s_{i}(t)$ and $f_{i}$ are the $i$-th primary user's baseband signal and carrier frequency, respectively. $s_{i}(t)$ is generated from filtering a white Gaussian noise with low pass filter $h(t)$. The pass-band width, or baseband signal bandwidth, of $h(t)$ is $B . M$ is the number of primary users. $n(t)$ is the additive white Gaussian noise. Simulation conditions are set as follows: 1 . The bandwidth of the band-limited WSS signal is $1 \mathrm{GHz}$. 2. The number of primary users is $M=5$. The baseband signal bandwidth is $B=50 \mathrm{MHz}$. Primary users' carrier frequencies are chosen from spectrum cells' center frequencies. 3. Baseband signal $s_{i}(t) \sim \mathcal{N}\left(0, \sigma_{s}^{2}\right)$ and additive white Gaussian noise $n(t) \sim \mathcal{N}\left(0, \sigma_{n}^{2}\right)$, where $\sigma_{n}^{2}$ is fixed and $\sigma_{s}^{2}$ is scaled to a certain SNR level. The noise floor is $-114 \mathrm{dBm}$. The false probability is $\mathrm{PF}=0.01$. 4. The Nyquist rate is $f_{N}=2 \mathrm{GHz}$, the ADC's sampling rate is $f_{A D C}=f_{N} / 4$. 5. The MCS parameter $L=24, q=6$ and sampling pattern $C=\{1,2,9,12,14,18\} .6$. To simulate the mismatch behaviour of parallel MCS, the clock timing skew is $2 \%$ of $T$. The offset and gain mismatch are $2 \%$ and $2 \%$ of signal's maximal amplitude, respectively.
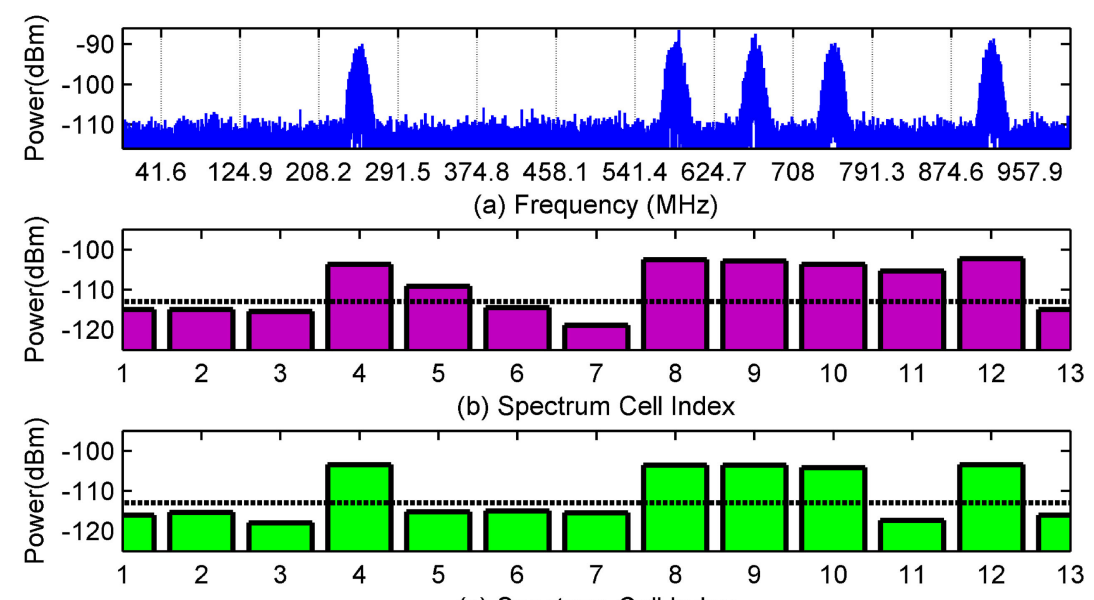

(c) Spectrum Cell Index

Fig. 4. Estimated finite resolution PSD comparision. (a) Original PSD.

(b) The results estimated by the traditional parallel MCS.

(c) The results estimated by the proposed serial MCS.

In the first simulation, we tested the feasibility of spectrum sensing using the proposed serial MCS. The SNR of the test signal is $10 \mathrm{~dB}$, and the number of samples per coset $N_{0}$ is 1000. Fig. 4(a) shows the PSD of the test signal. Fig. 4(b) and Fig. 4(c) give the results of finite-resolution PSD estimation based on the traditional parallel MCS and the proposed serial MCS, respectively. The dashed black lines in Fig. 4(b) and Fig. 4(c) represent the primary user detection threshold. The actual number of primary users in Fig. 4(a) is 5, and the number of spectrum cells exceeding the threshold in Fig. 4(b) and Fig. 4(c) is 7 and 5, respectively. In Fig. 4(b), there is a large error in the estimation of the PSD corresponding to the fifth and eleventh spectrum cell, thus these two spectrum cells are mistakenly considered occupied. The large error in the PSD estimation of these two spectrum cells is caused by the mismatch among sub-ADCs in parallel MCS. The mismatch affects the estimation accuracy of covariance matrix, and thus affects the estimation 
accuracy of the finite resolution PSD. As shown in Fig. 4(c), the proposed serial MCS can achieve higher estimation accuracy.

In the second simulation, we evaluate the detection performance of the proposed spectrum sensing method by calculating the probability of detecting the primary user as

$$
P_{d}=\frac{1}{M} \sum_{i=1}^{M}\left[\left(\hat{X}_{l i}-\gamma\right)>0\right],
$$

and the false alarm probability as

$$
P_{f}=\frac{1}{L-M} \sum_{j=1}^{L-M}\left[\left(\hat{X}_{l j}-\gamma\right)>0\right],
$$

where $l i$ and $l j$ are the index of the $i$-th primary user and $j$-th spectrum hole, respectively, $\hat{X}_{l i}$ and $\hat{X}_{l j}$ are the estimated averaged PSD in the $l i$-th and $l j$-th spectrum cell, respectively, and $\gamma$ is the detection threshold. The SNR is chosen to be $-3 \mathrm{~dB}, 0 \mathrm{~dB}$ and $3 \mathrm{~dB}$. The number of samples varies from 100 to 2000. 200 trials are performed and the averaged results are shown in Fig. 5 and Fig. 6.

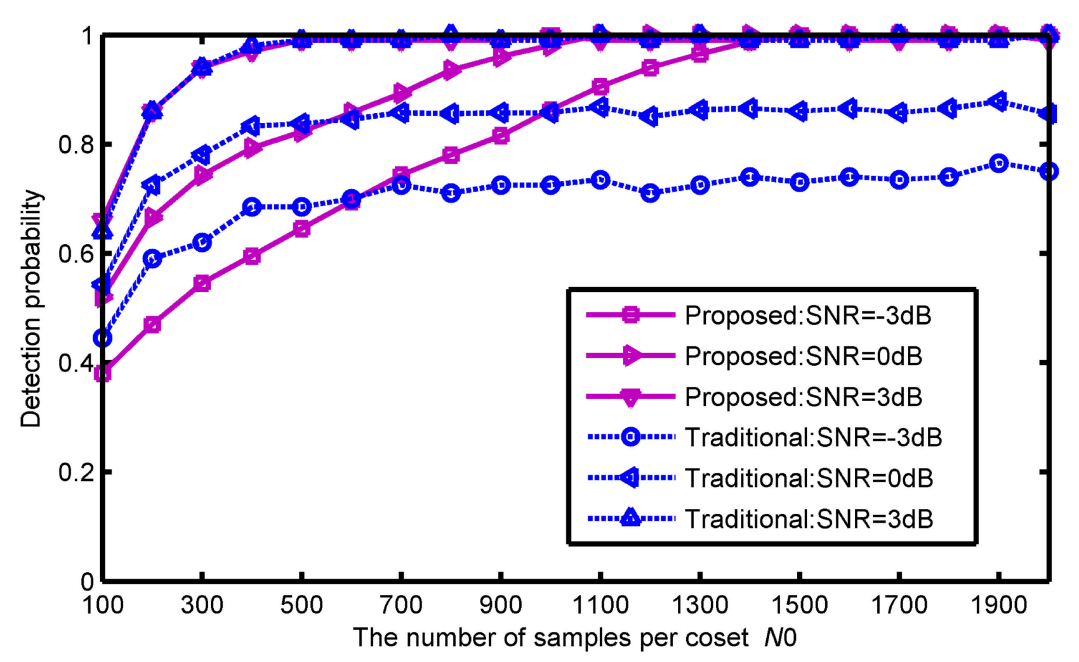

Fig. 5. Detection probability of the primary user.

As shown in Fig. 5, in the serial MCS, the detection probability increases as the number of samples per coset $N_{0}$ increases, and the primary user can always be detected with a high probability when the number of samples is large enough in the case of different SNR. However, in the parallel MCS, when the SNR is $0 \mathrm{~dB}$ and $-3 \mathrm{~dB}$, the detection probability does not increase after reaching 0.85 and 0.75 with increasing $N_{0}$. When $N_{0}$ is less than 600 , the detection probability of the parallel MCS is higher than that of the serial MCS for the same SNR. The reason is that the error signal generated by the mismatch in parallel MCS is mistakenly considered to be the primary user, so that the detection probability of the primary user is relatively high. In the proposed serial MCS, for signals with SNR higher than $-3 \mathrm{~dB}$, the primary user can be detected with a high probability when the number of samples per coset $N_{0}$ is greater than 1400 .

Compared with the detection probability, the false alarm probability is also very important. False alarm probability determines how many spectrum holes are 
available to secondary users. As shown in Fig. 6, in the proposed serial MCS, the false probability decreases with increasing the number of samples per $\operatorname{coset} N_{0}$. The reason is that the sample covariance is closer to the population covariance with increasing $N_{0}$. At the same time, in the traditional parallel MCS, the false alarm probability gradually increases with increasing $N_{0}$. The reason is that the sample covariance's estimation error, due to the mismatch among sub-ADCs, accumulates with increasing $N_{0}$. In the proposed serial MCS, the false alarm probability is almost zero for signals with SNR higher than $-3 \mathrm{~dB}$ when the number of samples per coset $N_{0}$ is greater than 1400. In other words, all spectrum holes can be found with a high probability.

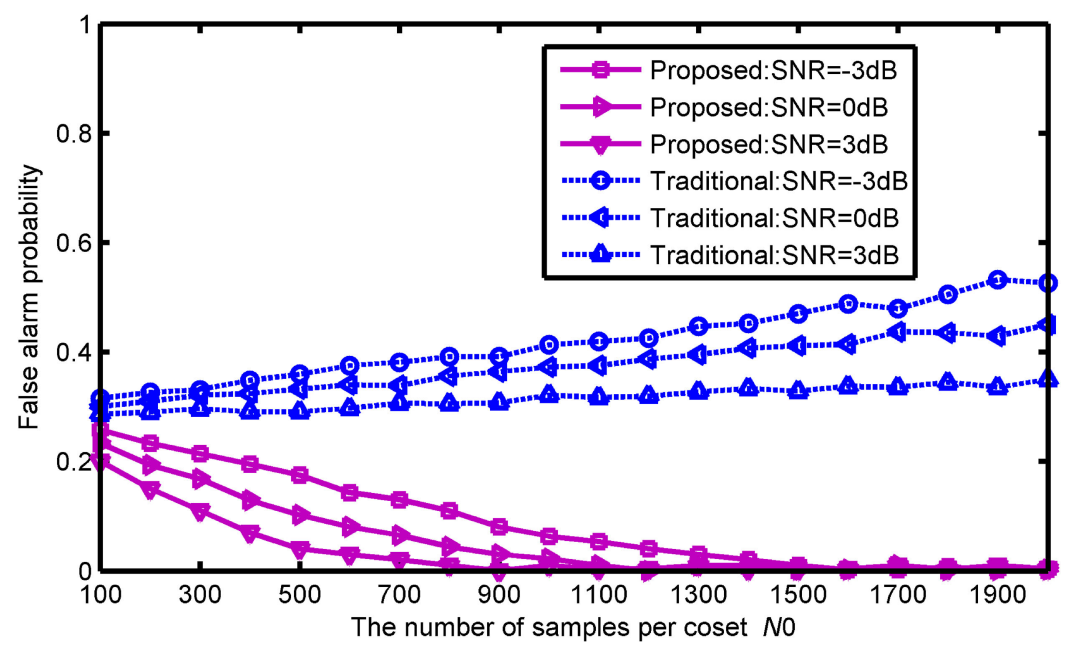

Fig. 6. False alarm probability of the primary user.

\section{Conclusion}

This letter proposes a single channel sub-Nyquist sampling structure, serial MCS, for wideband spectrum sensing. Although the requirement for the sampling rate of ADC is higher compared with the traditional parallel MCS, the proposed serial MCS can not only avoid the mismatch among sub-ADCs in traditional parallel MCS but also reduce the hardware implementation size. The sampling pattern design and a five-step spectrum sensing method are presented as well. Simulation results show that the mismatch among sub-ADCs seriously deteriorates the spectrum sensing performance in the traditional parallel MCS, and the proposed serial MCS exhibits outstanding detection performance for signals with SNR higher than $-3 \mathrm{~dB}$ when the number of samples per coset is greater than 1400 .

\section{Acknowledgments}

This work was supported by the National Nature Science Foundation of China (No. 61701077 and No. 61501087) and the Fundamental Research Funds for the Central Universities of China (No. ZYGX2016J100). 